\title{
CONSTRUÇÃO ARTESANAL DE UM ELETRODO ÍON SELETIVO A CHUMBO(II): UMA ALTERNATIVA PARA DISCIPLINAS EXPERIMENTAIS
}

\author{
Jonatas Gomes da Silva, Arilson Lehmkuhl e Silvia Keli de Barros Alcanfor* \\ Curso de Química, Universidade Católica de Brasília, QS 07 lote 01 EPCT Águas Claras, 71966-700 Taguatinga - DF, Brasil
}

Recebido em 1/9/07; aceito em 28/8/08; publicado na web em 5/2/09

\begin{abstract}
HOMEMADE CONSTRUCTION OF A LEAD(II) ION SELECTIVE ELECTRODE : AN ALTERNATIVE TO EXPERIMENTAL SUBJECTS. This works proposes a homemade construction of a lead(II) ISE of solid membrane $\left(\mathrm{Ag}_{2} \mathrm{~S} / \mathrm{PbS}\right)$ to determine $\mathrm{Pb}^{2+}$ ions in potentiometric titrations, using damaged combined glass electrodes. This electrode can be constructed in teaching laboratories, using it as a learning tool related to the theoretical principle of ISE. The analytical curve obtained $(y=27.056 x+337.58 ; R=$ 0.996) was linear on the range of $1 \times 10^{-5}$ to $1 \mathrm{~mol} \mathrm{~L}^{-1}$ and has presented a very close behavior of the Nernstian. The homemade ISE has presented a similar selectivity to the commercial electrodes, showing to be a good alternative to the experimental activities on teaching laboratories.
\end{abstract}

Keywords: ion selective electrode; lead; potentiometric titration.

\section{INTRODUÇÃO}

Os eletrodos íon-seletivos (EIS) são sensores eletroquímicos que permitem a determinação potenciométrica da atividade de uma espécie iônica na presença de outros íons,,$^{1,2}$ sendo utilizados em análises clínicas, medindo a atividade dos íons $\mathrm{K}^{+}, \mathrm{Na}^{+}, \mathrm{Ca}^{2+}, \mathrm{e} \mathrm{Cl}^{-}$no sangue, soro e urina, ${ }^{3} \mathrm{em}$ análises ambientais no monitoramento de metais ${ }^{4} \mathrm{e}$ em várias outras análises.

Durante muitos anos as aplicações dos EIS foram limitadas devido aos altos limites de detecção e à baixa seletividade. No entanto, este cenário mudou recentemente com a possibilidade de construir EIS com baixos limites de detecção, tornando-se desta forma viável em aplicações analíticas. Os recentes avanços e as novas perspectivas dos EIS foram relatadas por Torres e colaboradores. ${ }^{5}$

Dos EIS o mais utilizado é o eletrodo de $\mathrm{pH}$ (seletivo a íons $\mathrm{H}_{3} \mathrm{O}^{+}$), comercializado como eletrodo de vidro combinado (EVC), onde o eletrodo de membrana de vidro e o de referência estão em um único corpo e a fina membrana de vidro, na forma de um bulbo na parte inferior do eletrodo, é a parte sensível aos íons $\mathrm{H}_{3} \mathrm{O}^{+}$. A membrana de vidro pode ser substituída ou modificada por monocristais, como no eletrodo seletivo a fluoreto no qual é utilizado um cristal de fluoreto de lantânio ou por membrana sólida de disco prensado, a qual pode ser composta por trocador de íons orgânico ou de sulfeto de prata. ${ }^{6}$ Nesses eletrodos de membrana sólida, o contato elétrico pode ser líquido ou sólido. No contato líquido, tem-se no interior do eletrodo uma solução contendo o íon do metal de interesse e um eletrodo de referência interno. No caso do contato sólido, a membrana é colada diretamente ao fio condutor. ${ }^{7}$

A literatura cita a aplicação de eletrodos construídos com membrana sólida de disco prensado compostas por sulfetos na determinação dos íons $\mathrm{Pb}^{2+}, \mathrm{Cd}^{2+} \mathrm{e} \mathrm{Cu}^{2+}$, a depender da sua composição, além de sulfeto $\left(\mathrm{S}^{2-}\right)$ e $\mathrm{Ag}^{+} .{ }^{-11}$

Nas disciplinas experimentais de Química, a potenciometria é geralmente abordada com ênfase em titulações potenciométricas ácido-base e oxi-redução. A facilidade de aquisição do EVC para medidas de $\mathrm{pH}$ e do eletrodo de platina para acompanhar reações de oxi-redução, aliada à dificuldade de aquisição de EIS, devido ao seu

*e-mail: alcanfor@pos.ucb.br alto custo, reforçam esta tendência. No entanto, com o aumento da pesquisa na área de sensores potenciométricos e, conseqüentemente, a utilização destes sensores em análises químicas, faz-se necessária a introdução de EIS nos laboratórios de ensino de graduação. Dessa forma, é possível discutir os aspectos teóricos pertinentes, permitindo assim a familiarização do estudante com esta ferramenta analítica em ascendência nos últimos anos. Uma vez de posse de EIS, estes poderiam ser utilizados no monitoramento de titulações de precipitação e de complexação.

Neste contexto propõe-se a utilização do EVC danificado na construção de um EIS a chumbo(II) para ser utilizado como material didático, nos laboratórios de ensino, em titulações potenciométricas na determinação de íons $\mathrm{Pb}^{2+}$ com EDTA.

\section{PARTE EXPERIMENTAL}

\section{Reagentes e soluções}

Sulfeto de sódio $\left(\mathrm{Na}_{2} \mathrm{~S} .9 \mathrm{H}_{2} \mathrm{O}\right)$ (Labsynth), com teor de sulfeto (ca. 70\%) determinado experimentalmente por meio do método iodométrico, ${ }^{12}$ para a confecção da membrana sólida.

Para a obtenção da curva analítica foram preparadas soluções padrão com o sal de nitrato de chumbo (Isofar) e para o estudo de interferentes, soluções com os sais de nitrato de cobre (Vetec) e nitrato de prata (Cennabras). Todos os reagentes utilizados foram de grau analítico (PA), e sem purificação prévia. As soluções foram preparadas com água deionizada.

Foi utilizada água mineral Bonaqua ${ }^{\circledR}$ (fabricada pela Coca-Cola, purificada, gaseificada e por processo industrial adicionados os seguintes sais: cloreto de cálcio $2,52 \times 10^{-4} \mathrm{~mol} \mathrm{~L}^{-1}$; sulfato de magnésio $1,56 \times 10^{-4} \mathrm{~mol} \mathrm{~L}^{-1}$; bicarbonato de sódio $1,42 \times 10^{-4} \mathrm{~mol} \mathrm{~L}^{-1}$; cloreto de sódio $8,55 \times 10^{-6} \mathrm{~mol} \mathrm{~L}^{-1}$ e cloreto de potássio $6,71 \times 10^{-6} \mathrm{~mol} \mathrm{~L}^{-1}$ ), na preparação de uma solução enriquecida com chumbo para análise.

\section{Confecção da membrana sólida}

A membrana sólida utilizada na construção do EIS a chumbo(II) foi preparada por meio da precipitação do sulfeto de prata $\left(\mathrm{Ag}_{2} \mathrm{~S}\right)$ e do sulfeto de chumbo $(\mathrm{PbS})$, seguindo o procedimento descrito 
por Heijne e colaboradores. ${ }^{13} \mathrm{Na}$ precipitação da mistura de sulfetos, foram adicionados $80 \mathrm{~mL}$ da solução de sulfeto de sódio 1 mol L ${ }^{-1}$ (100\% de excesso) em $100 \mathrm{~mL}$ de solução contendo 0,056 mol de nitrato de prata $\left(\mathrm{AgNO}_{3}\right)$ e 0,012 mol de nitrato de chumbo $\left(\mathrm{Pb}\left(\mathrm{NO}_{3}\right)_{2}\right)$, para obter um precipitado com razão de $70 \%$ de sulfeto de prata $\left(\mathrm{Ag}_{2} \mathrm{~S}\right)$ e $30 \%$ de sulfeto de chumbo $(\mathrm{PbS})$. Esta razão de sulfetos é a que apresenta uma melhor propriedade eletroquímica. ${ }^{13}$ A solução contendo a mistura foi aquecida até $75^{\circ} \mathrm{C}$ e o precipitado obtido foi lavado quatro vezes com água deionizada, uma vez com solução de ácido nítrico $0,1 \mathrm{~mol} \mathrm{~L}^{-1}$, três vezes com água deionizada e três vezes com acetona, após a lavagem o precipitado foi seco a vácuo durante uma semana.

Para a moagem do precipitado foi necessário montar um moinho para a obtenção de sólidos com granulação inferior a $0,125 \mathrm{~mm}$. Nesta montagem, a cabeça de porcelana de um pistilo foi adaptada a um agitador mecânico (Quimis) e a moagem foi realizada em um gral de porcelana.

Após a moagem do precipitado, a membrana sólida foi confeccionada aplicando uma força de $2 \mathrm{t}$ utilizando uma prensa hidráulica (schulz) sob aquecimento com maçarico (oxigênio/acetileno, temperatura entre 1000 e $1400^{\circ} \mathrm{C}$ ), onde o precipitado permaneceu por um período de $60 \mathrm{~min}$. Ao final do processo obteve-se uma membrana com $0,8 \mathrm{~mm}$ de espessura e 5,85 $\mathrm{mm}$ de diâmetro.

\section{Construção do eletrodo}

Na construção do EIS a chumbo(II), foi utilizado um eletrodo combinado de $\mathrm{pH}$ (Quimis) com o eletrodo de referência danificado, não podendo mais ser utilizado em medidas potenciométricas de pH. Constatada a integridade elétrica dos contatos do eletrodo de $\mathrm{pH}$, o bulbo foi removido e a solução interna escoada. Para efetuar o contato elétrico, a membrana sólida confeccionada foi colada no fio de prata interno do eletrodo (fio condutor), utilizando uma mistura de araldite (adesivo epóxi) e grafite. O eletrodo foi vedado com cola de silicone (Figura 1).

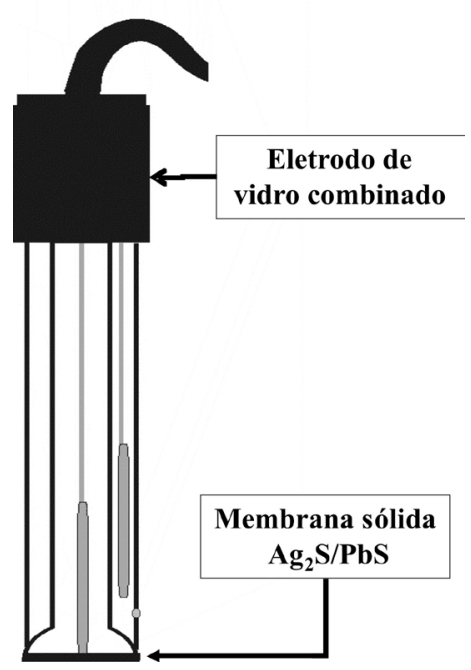

Figura 1. Construção do EIS a chumbo(II) artesanal

\section{Obtenção da curva analítica}

Para a obtenção da curva analítica foram preparadas soluções padrão de $\mathrm{Pb}^{2+}$ no intervalo de $10^{-7}$ até $1 \mathrm{~mol} \mathrm{~L}^{-1}$, e para a aquisição dos dados foi utilizado um potenciômetro modelo DM 20 (Digimed) com precisão de $0,1 \mathrm{mV}$ e um eletrodo de referência $\mathrm{Ag} / \mathrm{AgCl}$ modelo DME - R11 (Digimed).

\section{Aplicação do eletrodo}

O eletrodo artesanal foi usado como eletrodo indicador na determinação potenciométrica de íons $\mathrm{Pb}^{2+}$ em água deionizada e em água mineral Bonaqua ${ }^{\circledR}$ enriquecida com chumbo. Nesta determinação foi utilizado o método complexométrico com solução de EDTA $1 \times 10^{-2} \mathrm{~mol} \mathrm{~L}^{-1}$ e o $\mathrm{pH}$ mantido no intervalo de 4-6. O sistema para a determinação potenciométrica de íons $\mathrm{Pb}^{2+}$ foi montado utilizando um potenciômetro modelo DM 20 (Digimed), um eletrodo de referência $\mathrm{Ag} / \mathrm{AgCl}$ (Digimed) e o eletrodo artesanal de íon seletivo a chumbo(II) (Figura 2). A titulação potenciométrica foi conduzida e a variação de ddp $(\mathrm{mV})$ foi monitorada em função da formação do complexo entre o EDTA e o íon $\mathrm{Pb}^{2+}$.

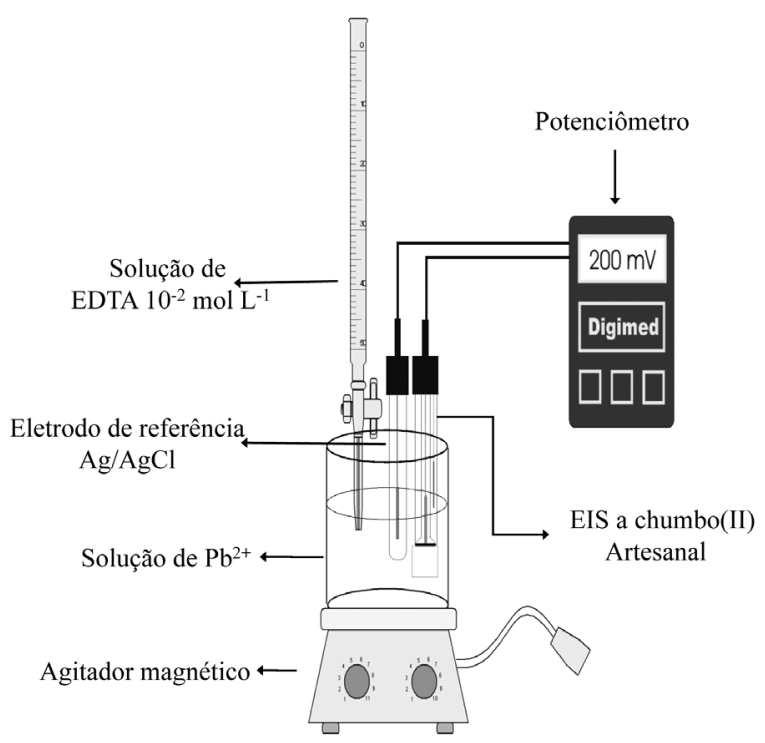

Figura 2. Sistema utilizado para a determinação potenciométrica de íons $\mathrm{Pb}^{2+}$

\section{RESULTADOS E DISCUSSÃO}

\section{Curva analítica}

A Figura 3 mostra a curva analítica obtida com EIS a chumbo(II) artesanal com soluções padrão sem ajustar a força iônica. O eletrodo seletivo artesanal respondeu de maneira linear ao logaritmo da atividade de $\mathrm{Pb}^{2+}$ no intervalo de concentração de $1 \times 10^{-5}$ a $1 \mathrm{moL} \mathrm{L}^{-1}$, com inclinação angular $27,05 \mathrm{mV} \mathrm{dec}^{-1}(\mathrm{R}=0,996)$, um comportamento próximo do nernstiano, onde o valor esperado era de $29,58 \mathrm{mV} \mathrm{dec}{ }^{-1}$, para $\mathrm{T}=25^{\circ} \mathrm{C}$, segundo a Equação de Nernst $\left(\mathrm{E}=\mathrm{E}^{\mathrm{o}}+\frac{59,16}{\mathrm{n}} \log \mathrm{a}_{\mathrm{M}^{\mathrm{n}}}\right)$. O eletrodo artesanal apresentou boa reprodutibilidade, um tempo de resposta menor que 30 s e não apresentou efeito de memória nos ensaios realizados.

Os EIS respondem à atividade dos íons em análise e como normalmente se deseja conhecer concentrações e não atividades é comum o uso de sal inerte para levar todas as soluções padrões e a amostra a uma força iônica alta e constante. Se os coeficientes de atividade permanecem constantes, o potencial do eletrodo fornece concentrações diretamente. ${ }^{14} \mathrm{O}$ mesmo procedimento para obtenção da curva analítica foi realizado com soluções padrões com força iônica alta e constante (adição do sal inerte $\mathrm{KNO}_{3} 0,1 \mathrm{~mol} \mathrm{~L}^{-1}$ ). Os resultados foram semelhantes aos obtidos com soluções sem o ajuste da força iônica, indicando que a resposta deste eletrodo, dentro da faixa de concentração estudada, sofre ação mínima da força iônica do meio. 


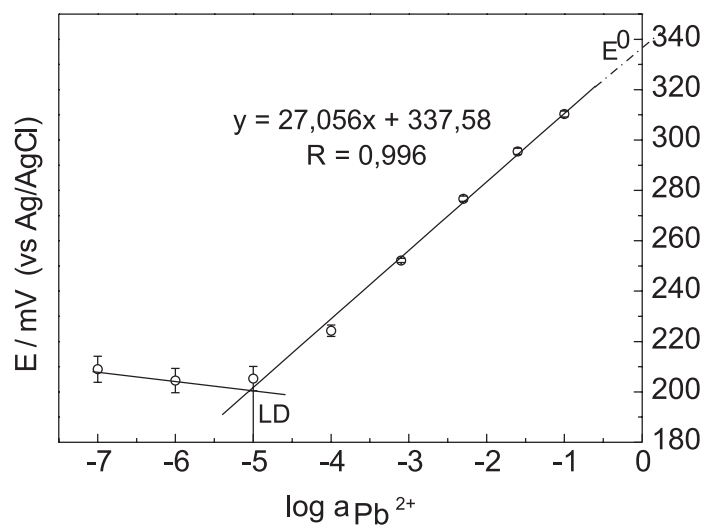

Figura 3. Curva analítica do EIS a chumbo(II) artesanal $(n=6)$

O potencial padrão $\left(\mathrm{E}^{0}\right)$ do eletrodo artesanal foi obtido por extrapolação ${ }^{11}$ para $\log \mathrm{a}_{\mathrm{Pb}}{ }^{2+}=0$ (Figura 3), obtendo um valor de $337,58 \mathrm{mV}$ vs $\mathrm{Ag} / \mathrm{AgCl}$.

\section{Limite de detecção}

O limite de detecção (LD), definido como sendo a menor concentração do íon primário presente em uma amostra que pode ser detectado sob as condições experimentais estabelecidas, foi de aproximadamente $1 \times 10^{-5} \mathrm{~mol} \mathrm{~L}^{-1}$ para o EIS a chumbo(II) artesanal. Este foi determinado, de acordo com a recomendação da IUPAC, ${ }^{15}$ por meio da intersecção da extrapolação das duas retas da curva analítica (Figura 3).

\section{Seletividade}

Como os EIS não respondem exclusivamente, em toda a faixa de concentração, a um tipo de íon, é necessário fazer um estudo dos íons interferentes. Na potenciometria este estudo é feito por meio da determinação dos coeficientes de seletividade potenciométricos $\left(\mathrm{K}^{\mathrm{POT}}\right)$ pelos seguintes métodos: método do interferente fixo (MIF), que consiste na medida de uma solução com concentração fixa do íon interferente, variando a concentração do íon primário e o método das soluções separadas (MSS), que envolve a medida de duas soluções, onde cada solução contém só um íon de interesse. ${ }^{16-18}$

Neste estudo de interferentes a resposta do EIS a chumbo(II) foi medida em função do logaritmo das atividades dos íons $\mathrm{Cu}^{2+} \mathrm{e}$ $\mathrm{Ag}^{+}$. Os dados obtidos nessas medidas foram colocados na forma de gráfico (Figura 4), com o intuito de facilitar a compreensão dos estudantes, permitindo a determinação dos íons interferentes, sem fazer cálculos.

Observa-se na Figura 4, que os íons $\mathrm{Cu}^{2+}$ e $\mathrm{Ag}^{+}$são interferentes na determinação de $\mathrm{Pb}^{2+}$, pois para estes íons o eletrodo artesanal apresentou um potencial maior que o obtido para o íon primário. Portanto, o eletrodo artesanal também pode ser utilizado na determinação do íon $\mathrm{Cu}^{2+}$ no intervalo onde o comportamento do eletrodo foi linear, lembrando que este eletrodo será utilizado apenas para fins didáticos.

Avançando um pouco mais na percepção do estudante, calculouse o valor de $\log \mathrm{K}^{\text {pot }}$ de 27,45 para os íons $\mathrm{Cu}^{2+}$, utilizando o MSS por meio da seguinte equação:

$$
\log \mathrm{K}_{\mathrm{A}, \mathrm{B}}^{\mathrm{pot}}=\frac{\mathrm{E}_{\mathrm{B}}-\mathrm{E}_{\mathrm{A}}}{2303 \mathrm{RT} / \mathrm{Z}_{\mathrm{A}} \mathrm{F}}+\left(1-\frac{\mathrm{Z}_{\mathrm{A}}}{\mathrm{Z}_{\mathrm{B}}}\right) \log \mathrm{a}_{\mathrm{A}}
$$

onde: $\mathrm{E}_{\mathrm{A}}$ e $\mathrm{E}_{\mathrm{B}}=$ potencial observado experimentalmente para o íon primário (A) e para o íon interferente $(\mathrm{B}) ; \mathrm{R}=$ constante dos gases

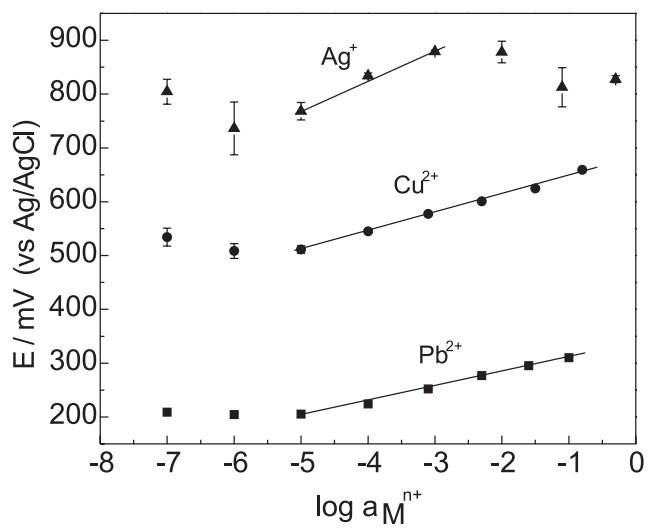

Figura 4. Estudo dos interferentes para o EIS a chumbo(II)

$\left(8,314 \mathrm{~J} \mathrm{~K}^{-1} \mathrm{~mol}^{-1}\right) ; \mathrm{T}=$ temperatura em kelvin; $\mathrm{F}=$ constante de Faraday $\left(96500 \mathrm{C} \mathrm{mol}^{-1}\right) ; \mathrm{Z}_{\mathrm{A}}$ e $\mathrm{Z}_{\mathrm{B}}=$ carga do íon primário (A) e do íon interferente $(\mathrm{B}) ; \mathrm{a}_{\mathrm{A}}=$ atividade do íon primário.

Quanto maior o valor do $\mathrm{K}^{\text {pot }}$ maior a interferência do íon $\mathrm{B}$, portanto, pode-se concluir que os íons $\mathrm{Cu}^{2+}$ interferem fortemente na medida do íon primário, como observado na Figura 4. O MSS é recomendado somente se o eletrodo exibir uma resposta nernstiana, inviabilizando assim a sua utilização para a obtenção do valor do $\mathrm{K}^{\text {pot }}$ para os íons $\mathrm{Ag}(\mathrm{I})$.

A Tabela 1 mostra os íons interferentes para um EIS a chumbo(II) comercial e para o artesanal. Neste trabalho não foi feito o estudo de interferência para os íons $\mathrm{Hg}^{2+}$. De qualquer forma, os íons $\mathrm{Ag}^{+}$, $\mathrm{Hg}^{2+} \mathrm{e} \mathrm{Cu}^{2+}$ devem estar ausentes nas medidas do íon primário, pois formam sulfetos menos solúveis $\left(\mathrm{Kps}_{\mathrm{Ag} 2 \mathrm{~S}}=8 \times 10^{-51} ; \mathrm{Kps}_{\mathrm{HgS}}=2 \mathrm{x}\right.$ $\left.10^{-53} \mathrm{e} \mathrm{Kps}_{\mathrm{CuS}}=8 \times 10^{-37}\right)$ que o chumbo ${ }^{19}\left(\mathrm{Kps}_{\mathrm{PbS}}=3 \times 10^{-28}\right)$.

Tabela 1. Interferentes para o eletrodo de íon seletivo a chumbo(II)

\begin{tabular}{lc}
\hline EIS a chumbo(II) & Interferentes \\
\hline Comercial & $\mathrm{Hg}^{2+} ; \mathrm{Ag}^{+} ; \mathrm{Cu}^{2+}$ \\
Artesanal & $\mathrm{Ag}^{+} ; \mathrm{Cu}^{2+}$ \\
\hline
\end{tabular}

* Eletrodo de íon seletivo a chumbo(II) da Metrohm

\section{Aplicação}

O eletrodo artesanal é utilizado, como eletrodo indicador na titulação de íons $\mathrm{Pb}^{2+}$ com EDTA em água deionizada e em água mineral $\left(\right.$ Bonaqua $^{\circledR}$ ) enriquecida com chumbo em aulas experimentais de Química Analítica III (Métodos Eletroquímicos) do Curso de Química da Universidade Católica de Brasília, desde o ano de 2004. A Figura 5a mostra as curvas potenciométricas obtidas pelos estudantes na titulação de $100 \mathrm{~mL}$ de $\mathrm{Pb}^{2+} 1 \times 10^{-3} \mathrm{~mol} \mathrm{~L}^{-1}$ com EDTA $1 \times 10^{-2} \mathrm{~mol} \mathrm{~L}^{-1}$ em água deionizada e em água mineral Bonaqua ${ }^{\circledR}$. Observa-se na Figura 5a o decréscimo do potencial com a adição da solução de EDTA. As variações de potencial foram significativas para as curvas obtidas, indicando que o eletrodo pode ser utilizado na titulação potenciométrica de complexação de $\mathrm{Pb}^{2+}$.

Por meio das curvas obtidas da Figura 5a, nota-se um perfil semelhante na resposta do eletrodo para as duas amostras, portanto, os íons presentes na água mineral Bonaqua ${ }^{\circledR}$ não interferem na determinação potenciométrica de íons $\mathrm{Pb}^{2+}$. Para a obtenção do valor dos volumes de equivalência, foi utilizado o gráfico da primeira derivada (Figura $5 b)$, onde para as soluções em água deionizada e em água Bonaqua ${ }^{\circledR}$ obtiveram-se volumes de equivalência de 10,5 e 10,3 mL, respectivamente, valores bem próximos do teórico de $10 \mathrm{~mL}$. Desta forma, foi 

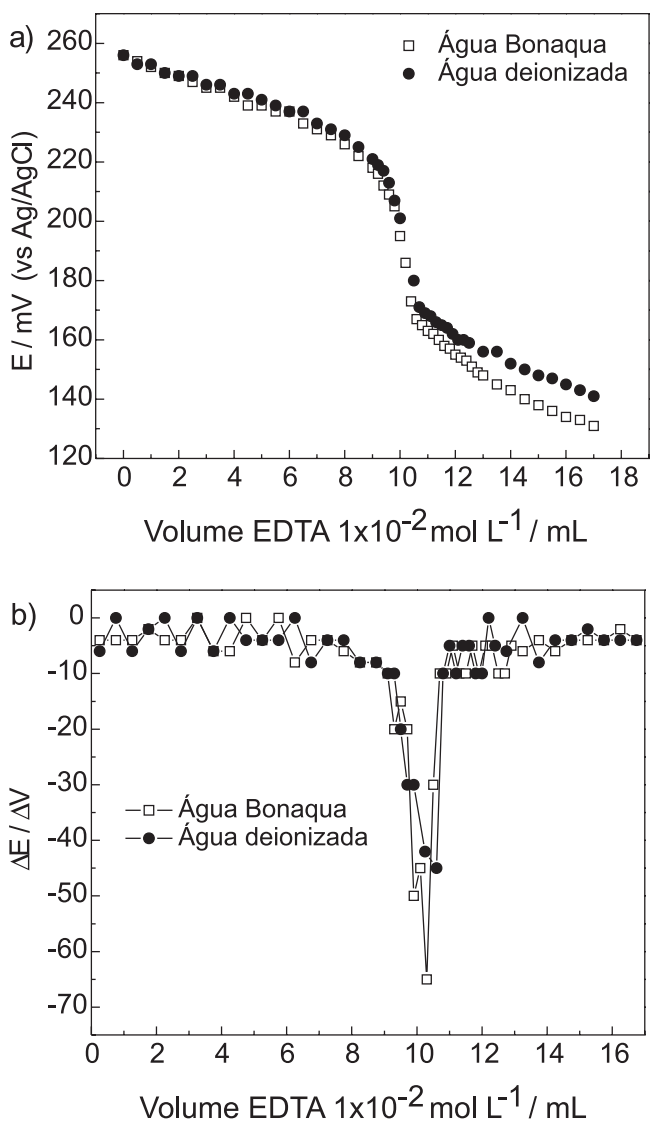

Figura 5. a) Determinação de íons $P b^{2+} 10^{-3} \mathrm{~mol} \mathrm{~L}^{-1}$ por complexometria com EDTA utilizando EIS a chumbo(II) artesanal. b) Primeira derivada da curva de titulação complexométrica

comprovada a aplicabilidade do eletrodo artesanal no monitoramento de íons $\mathrm{Pb}^{2+}$ em solução aquosa, podendo ser utilizado como uma ferramenta no ensino de eletroquímica.

\section{CONCLUSÃO}

O procedimento proposto para a construção artesanal do EIS a chumbo (II) é simples e de baixo custo, permitindo sua construção em laboratórios com poucos recursos, podendo ser aplicado em práticas de titulações potenciométricas em laboratórios de ensino. A construção e o uso do EIS proporcionaram a discussão de alguns conceitos importantes em química analítica como constante de solubilidade (Kps), atividade, força iônica, limite de detecção, seletividade e a Equação de Nernst, que foi utilizada para obtenção de curvas teóricas. Portanto, o uso do EIS artesanal mostrou-se como uma boa alternativa para atividades experimentais em laboratórios de ensino, ocasionando sem dúvida um ganho pedagógico para os estudantes.

\section{AGRADECIMENTOS}

Ao apoio financeiro da Universidade Católica de Brasília (UCB).

\section{REFERÊNCIAS}

1. Stradiotto, N. R.; Yamanaka, H.; Zanoni, M. V. B.; J. Braz. Chem. Soc. 2003, 14, 159.

2. Morf, W. E.; The Principles of Ion-Selective Electrodes and of Membrane transport, Elsevier: New York, 1981.

3. Bakker, E.; Pretsch, E.; Trends Anal. Chem. 2001, 20, 11.

4. Bakker, E.; Pretsch, E.; Anal. Chem. 2002, 74, 420A.

5. Torres, K. Y. C.; Marzal, P. C.; Kubota, L. T.; Quim. Nova 2006, 29, 1094.

6. Vogel, A. I.; Análise Química Quantitativa, 6ª ed., LTC: Rio de Janeiro, 2002.

7. Serrano, S. H. P.; Tese de Doutorado, Universidade de São Paulo, Brasil, 1988.

8. Skoog, A. D.; Holler, F. J.; Nieman,T .A.; Principles of Instrumental Analysis, $5^{\text {th }}$ ed., Saunders College Publishing: Orlando, 1998.

9. Cattrall, R. W.; Chemical sensors, Oxford: New York, 1997.

10. Koryta, J.; Anal. Chim. Acta 1972, 61, 329.

11. Koryta, J.; Anal. Chim. Acta 1977, 91, 1.

12. Eaton, A. D.; Standard Methods of Examination of Water \& Wastewater, $21^{\text {th }}$ ed., American Public Health Association: Washington, 2005.

13. Heijne, G. J. M.; Van Der Linden, W. E.; Den Boef, G.; Anal. Chim. Acta 1978, 100, 193

14. Harris, D. C.; Análise Química Quantitativa, $5^{\mathrm{a}}$ ed., LTC: Rio de Janeiro, 2001.

15. "Recommendations - 1975"; Pure Appl. Chem. 1976, 48, 127.

16. Back, R.; Linder, E.; Pure Appl. Chem. 1994, 66, 2527.

17. Umezawa, Y.; Umezawa, K.; Sato, H.; Pure Appl. Chem. 1995, 67, 507.

18. Umezawa, Y.; Bühlmann, P.; Umezawa, K.; Tohda, K.; Ameya, S.; Pure Appl. Chem. 2000, 72, 1851.

19. Mascini, M.; Liberti, A.; Anal. Chim. Acta 1972, 60, 405. 